\title{
Design of novel passive optical switching system using shared wavelength conversion with electrical buffer
}

\author{
Ji-Hwan Kim ${ }^{1}$, JungYul Choi ${ }^{2}$, Minho Kang ${ }^{1}$, \\ and J.-K. Kevin Rhee ${ }^{1 \mathrm{a})}$ \\ ${ }^{1}$ Optical Internet Research Center, Information and Communications University, \\ 119 Munjiro, Daejeon, Korea \\ ${ }^{2}$ BcN Business Unit, KT Corp.
}

463-1 Jeonmin-dong, Yusung-gu, Daejeon, Korea

a)rhee.jk@ieee.org

\begin{abstract}
We propose a novel optical switching system equipped with shared wavelength converters with electrical buffers. Simulation results show that the proposed system significantly reduces the blocking probability below $10^{-6}$ with low buffering delay. We present benefits of the proposed switching system compared with the conventional dedicated wavelength converter scheme in terms of blocking probability and average delay time. The proposed switching system is believed to be a technoeconomically feasible and implementable solution for optical packet/burst switching with current optical technologies.
\end{abstract}

Keywords: optical switching system, passive switching, electrical buffer, wavelength conversion, OPS, OBS

Classification: Photonics devices, circuits, and systems

\section{References}

[1] A. Bianco, E. Leonardi, M. Munafo, F. Neri, and W. Picco, "Design of optical packet switching networks," GLOBECOM '02, vol. 3, pp. 27522756, 2002.

[2] S. Verma, H. Chaskar, and R. Ravikanth, "Optical burst switching: a viable solution for terabit IP backbone," IEEE Network, vol. 14, no. 6, pp. 48-53, 2000.

[3] L. Dittmann, C. Develder, D. Chiaroni, F. Neri, F. Callegati, W. Koerber, A. Stavdas, M. Renaud, A. Rafel, J. Sole-Pareta, W. Cerroni, N. Leligou, L. Dembeck, B. Mortensen, M. Pickavet, N. L. Sauze, M. Mahony, B. Berde, and G. Eilenberger, "The European IST Project DAVID: A Viable Approach Toward Optical Packet Switching," IEEE J. Select. Areas Commun., vol. 21, no. 7, pp. 1026-1040, 2003.

[4] C. M. Assi, A. A. Shami, M. A. Ali, Z. Zhang, and X. Liu, "Impact of Wavelength Converters on the Performance of Optical Networks," Optical Networks Mag., vol. 3, pp. 22-30, 2002.

[5] H. Li and I. L. Thng, "Performance Analysis of a Limited Number of 
Wavelength Converters in an Optical Switching Node," IEEE Photon. Technol. Lett., vol. 17, pp. 1130-1132, 2005.

[6] K. C. Lee and V. O. K. Li, "A Wavelength-Convertible Optical Networks," J. Lightwave Technol., vol. 11, pp. 962-970, 1993.

[7] A. Tzanakaki, I. Zacharopoulou, and I. Tomkos, "Broadband building blocks [optical networks]," IEEE Circuits Devices Mag., vol. 20, pp. 3237,2004 .

\section{Introduction}

Optical packet switching (OPS) [1] and optical burst switching (OBS) [2] technologies have been the most forward-looking research areas in the optical networking community for the last two decades. Yet, it seems that few technologies look promising to achieve the industry goals to provide an ultimate bandwidth solution, up to peta-bits/second, with practical application requirements of performance, cost, power consumption, and form factor. This paper presents a structured engineering result for an optimal switch architecture design that may lead to a paradigm shift toward an earlier commercial implementation of OPS/OBS technologies.

OPS (also OBS) systems consist of two main essential hardware functions: switch fabric and buffering. Both functions may utilize passive and active technologies, as shown in Table I. Most of active technologies utilize semiconductor optical amplifiers (SOA) and wavelength converters (WC) that require complicated optical signal processing, high power consumption, complex control requirements, and premium device technologies. Passive technologies can overcome such drawbacks, but incapable of wavelength conversion that is important to provide the wavelength continuity in WDM network systems.

Table I. Technology classification for optical switching

\begin{tabular}{|c||c|c|}
\hline Technology & optical switch fabric & optical buffer \\
\hline \hline Passive & electro-optic, acouto-optic, & fiber delay line, \\
(no WC) & ultra-fast MEMS, & recirculating loop, \\
& $\lambda$-selective switch & slow-light photonic crystal \\
\hline Active & electrical switch with OEO, & electrical memory with OEO \\
(WC capable) & AWGR/WC, SOA array & bistable laser diode \\
\hline
\end{tabular}

The traditional switch architecture consists of 'active switches with passive buffers,' where the wavelength continuity is fully provided [3]. This design rule may have been the critical road block against practical applications due to drawbacks of high cost, high power, and poor blocking probability. This letter reports that 'Passive switches with Active buffers' is more effective in blocking probability improvement. Being capable of achieving extraordinary performance, the active buffers can be shared, which can dramatically reduce the system cost, power consumption, and form factor to achieve petabit switches. 


\section{Wavelength conversion in optical networks}

\subsection{Classification of wavelength conversion schemes}

A wavelength converter is a device that changes one input wavelength to a different outgoing wavelength. There are several types of WCs according to sharing, conversion range, and its location: dedicated converter vs. shared converter [4], full-ranged converter vs. limited-ranged converter [5], and shared-per-node (SPN) converter vs. shared-per-link (SPL) converter [6]. The dedicated wavelength converters (DWCs) improve blocking performance more than shared wavelength converters (SWCs). A sharing ratio of $25 \%$ in a SPN wavelength converter scheme has been reported to produce almost the same performance as with the full installation of DWCs [4]. In [6], it is indicated that the SPN wavelength converter is more efficient than the SPL due to an algorithm benefit. Limited-range wavelength converters can reduce system cost further and its performance can be comparable with full-range converters by applying a delicate scheduling algorithm. Note that most wavelength conversion technology is quite costly as much as optical-toelectrical-to-optical transponders.

\subsection{Transparency vs. opaque switching system}

In transparent optical switching systems, incoming optical packets are routed through an optical switch fabric without optical-to-electrical conversion. This offers transparency to a variety of bit rates and protocols. The transparent switching system can utilize most benefits of the optical technology, such as almost unlimited bandwidth support, low power, security, and so on. However, it is difficult to detect faults in optical data so that OAM (operation, administration, and maintenance) functions may not be easily supported [7]. On the other hand, an opaque optical switching system is based on either electrical switching fabric or optical switching fabric surrounded by opticalto-electrical-to-optical conversion. The opaque switching system offers inherently $3 \mathrm{R}$ (regeneration, retiming, reshaping), wavelength conversion, and bit-level monitoring, and also provides powerful OAM functions. The opaque system, however, currently has difficulty in increasing bit rate and requires high transmission cost. It is still controversial to choose either approach considering all aspects of pros and cons.

\section{Proposed optical switching system}

An economical optical switching system with commercially deployable performance can be achieved by shared electrical buffering. In Section 2, we discuss that a shared-per-node wavelength converter scheme is the most economical choice and some part with electrical modules can much improve blocking performance as well as OAM functions compared with a transparent all-optical system. Figure 1 shows the proposed optical switching system where the majority of packets are passively switched by a large-scale optical fabric switch module. 


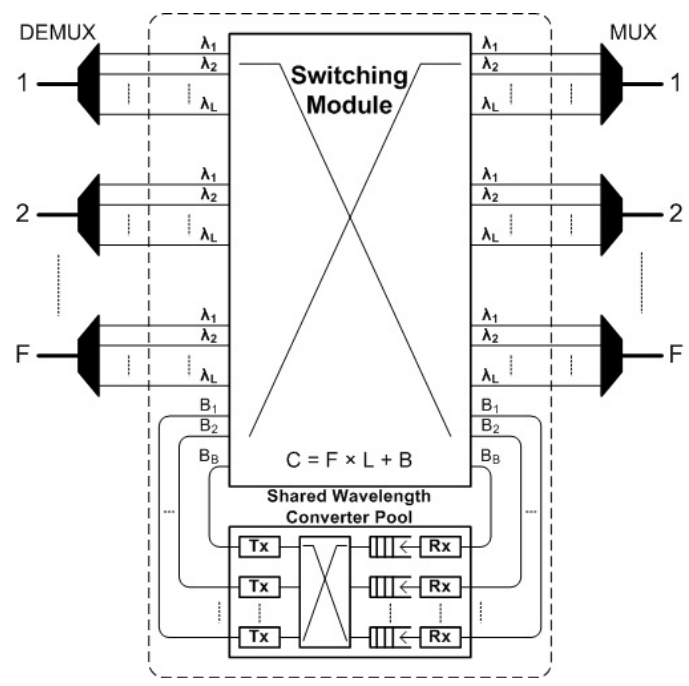

Fig. 1. Schematic node architecture of passive switching and shared active buffering

In the proposed system, WCs are shared with in a node which can resolve the wavelength continuity constraint. Thanks to sharing of costly WCs, the overall system cost can be greatly reduced. An SWC pool consists of opticalto-electrical converters (receivers), electrical buffers, an electrical switch, and electrical-to-optical converters whose output wavelength is rapidly tunable (transmitters capable of wavelength conversion). The overall optical switching system is equipped with $F$ input/output fiber links, $L$ wavelengths per link, and $B$ shared tunable WCs. Therefore, the number of total input ports of the optical switch module (C) is $F \times L+B$. The main advantages of the proposed optical switching system are significant reductions in blocking probability by using electrical buffers and the overall system cost by sharing costly components.

Now, let us explain how an optical packet is switched and transmitted through the switching system without contention. An optical packet from an input wavelength is switched and forwarded to the identical output wavelength at the destination output. This approach eliminates the requirement of wavelength converters in the switch module; otherwise, they would have been required for all wavelength channels of all links. When two or more optical packets from different input links at the same wavelength attempt to be transmitted toward the same destination output link simultaneously, contention occurs and subsequently the corresponding packets may be discarded.

Using the proposed optical switching system, however, contended packets are forwarded to the SWC pool if any input at the SWC is idle. An optical packet entering the SWC pool is converted from an optical signal to an electrical signal by a photo detector at a receiver. The converted packet is stored in the electrical buffer until one of wavelengths at the destination output link becomes idle. The packet is then forwarded to an available transmitter (e.g. tunable laser diode) which tunes to the idle output wavelength. Finally, the optical packet is switched to the idle wavelength of the destination output 
link through the optical switching module.

\section{Performance evaluation}

Benefits of the SWCs are presented in terms of blocking performance and buffering delay time under the following simulation parameters: the number of input/output fiber links (F) is 8, per-wavelength bandwidth $10 \mathrm{Gbps}$, the average optical packet size 100 kbytes with an exponential distribution. The arrival rate of packets with a Poisson process is normalized to ensure the offered load per wavelength to be between zero and one. Optical packets are assumed to be uniformly distributed to all output links. The electrical buffer is assumed to have a large enough depth of buffering, so there is no buffer overflow.

\subsection{Blocking probability}

Blocking probability in the proposed optical switching system is shown in Fig. 2 (a). The larger the sharing ratio of WCs with buffering capability, the lower the blocking probability can be achieved. For a target blocking probability $10^{-6}$, we can provision an offered load of 0.52 by use of $75 \%$ sharing ratio of WCs with respect to the total number of input wavelengths, i.e. $\mathrm{B} /(\mathrm{F} \times \mathrm{L})$ as shown in Fig. $2(\mathrm{a}-1)$, where the number of wavelengths

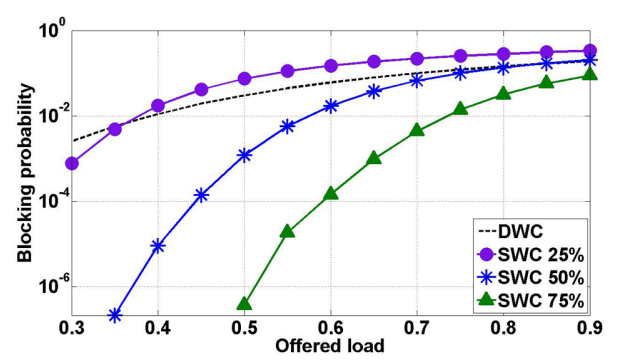

(a-1) For different sharing ratio of WC (W: 8$)$

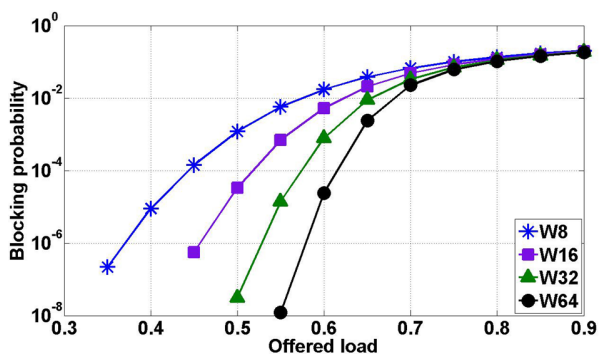

(a-2) For different number of wavelength (The sharing ratio of SWC is 50\%)

(a) Blocking probability

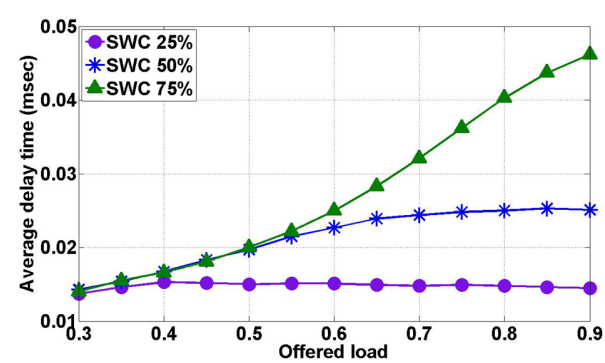

(b-1) For different sharing ratio of $\mathrm{WC}(\mathrm{W}: 8)$

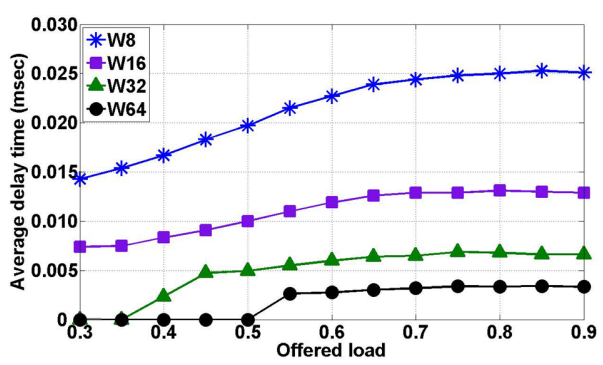

(b-2) For different number of wavelengths (the sharing ratio of SWC is $50 \%$.)

(b) Buffering delay time (The average packet length is $0.08 \mathrm{~ms}$.)

Fig. 2. Performance evaluation (Blocking probability, Buffering delay time.)

(c) IEICE 2006

DOI: 10.1587/elex.3.546 Received November 16, 2006 
(W) per link is 8 . This result is much better than the optical switching system with dedicated wavelength converters at all wavelengths. For example, $50 \%$ or more sharing ratio of WCs provides lower blocking probability than that with DWCs over all range of offered load. In addition, as the number of wavelengths per link increases, the performance benefit of the proposed optical switching system increases as shown in Fig. 2 (a-2).

\subsection{Buffering delay time}

Figure 2 (b) presents the average delay time of packets that is experienced at the electrical buffers. As the offered load increases, the delay time also increases, especially with a larger sharing ratio of SWCs as shown in Fig. 2 (b1). The reason is that more blocked packets attempt to access SWCs and are queued at the electrical buffers at a higher load. More SWCs can handle more blocked packets queued in the buffer, and subsequently they may lead to a longer delay time. On the other hand, with the same sharing ratio of SWCs, the increment in the number of wavelengths per link can reduce the buffering delay time as shown in Fig. 2(b-2), which is mostly due to low probability of contention.

Though packets experience buffering delay through electrical buffers, the impact of the delay is practically negligible. Even at the offered load 0.9 and $50 \%$ sharing ratio of SWCs with 8 wavelengths per link, the average delay time is just $25 \mu$ s so that delay sensitive applications can be well provisioned with satisfactorily low blocking probability.

\section{Conclusion}

We proposed a novel passive optical packet switching system design using shared wavelength converters with electrical buffers. Advantage of this system is that this scheme can achieve significant improvement in blocking probability below $10^{-6}$ at the practical offered load by use of only a half number of wavelength converters with respect to the number of input channels. Therefore, the proposed system can substantially reduce overall system cost and demonstrates possibilities to realize commercial optical switching systems.

\section{Acknowledgments}

This work was supported in part by the KOSEF-OIRC project. 